\title{
Knowledge, Adequacy, and Approximate Truth
}

Abstract: Approximation involves representing things in ways that might be close to the truth but are nevertheless false. Given the widespread reliance on approximations in science and everyday life, here we ask whether it is conceptually possible for false approximations to qualify as knowledge. According to the factivity account, it is impossible to know false approximations, because knowledge requires truth. According to the representational adequacy account, it is possible to know false approximations, if they are close enough to the truth for present purposes. In this paper, we adopt an experimental methodology to begin testing these two theories. When an agent provides a false and practically inadequate answer, both theories predict that people will deny knowledge. But the theories disagree about an agent who provides a false but practically adequate answer: the factivity hypothesis again predicts knowledge denial, whereas the representational adequacy hypothesis predicts knowledge attribution. Across two experiments, our principal finding was that people tended to attribute knowledge for false but practically adequate answers, which supports the representational adequacy account. We propose an interpretation of existing findings that preserves a conceptual link between knowledge and truth. According to this proposal, truth is not necessary for knowledge, but it is a feature of prototypical knowledge.

Keywords: truth; knowledge; approximation; practical interests; theory of mind 
All exact science is determined by the idea of approximation. - Bertrand Russell

\section{Introduction}

From everyday judgments to complex policy decisions to sophisticated scholarly inquiry, human beings ubiquitously rely on approximation. We approximate what temperature the coffee is safe to drink at, or the time needed to arrive at work, for example, to avoid becoming scalded or fired. Scientists and engineers rely on approximation when computing significant figures such as the decimals of pi to calculate accurate distances, or determining tradeoffs in computer programing to build useful algorithms (Mittal 2016; NASA/JPLedu 2016). These approximations are adequate enough for different practical purposes even though they are, strictly speaking, false. The fact that we so often rely on false approximations to inform important decisions raises an important question: can they qualify as knowledge?

As reasonable as it may initially sound to count them as knowledge, a leading philosophical theory denies the possibility. According to this theory, only true things can be known - that is, knowledge is factive (e.g. Audi 1998; BonJour 2002; Chisholm 1989; Davidson 2001; Feldman 2003; Fumerton 2006; Greco 2010; Pritchard 2006; Sartwell 1992; Sosa 1991; Williamson 2000; Zagzebski 2009). Researchers in other fields, including psychology (e.g. Starmans and Friedman 2012) and education (e.g. Sandoval, Greene, and Bråten 2016), have accepted this assumption. If knowledge requires truth but practically adequate approximations are not true, then it is not possible to know them. For example, if an engineer says that a foundation is 9910 inches long but it is actually 9902 inches, then the engineer does not know how long the foundation is. To 
the extent that we rely on such approximations, factivity threatens skepticism that potentially undermines many of things we typically take ourselves and others to know.

In support of the factivity account, several lines of research suggest strong conceptual connections between knowledge and truth in ordinary social cognition. First, studies on adult mental representation show large effects of truth on knowledge attribution (Blouw, Buckwalter, and Turri Forthcoming; Nagel, Juan, and Mar 2013; Starmans and Friedman 2012; Turri 2013b, 2016c). Though these studies do not focus on the issue of factivity directly, they include false-belief controls that represent paradigmatic ignorance. In one set of studies, for example, researchers isolated kinds of luck that are compatible and incompatible with knowledge attribution (Turri, Buckwalter, and Blouw 2015). To do this, they compared cases involving agents who luckily get a true answer to agents who get a false answer. Although sometimes people tended to attribute knowledge to the former, they strongly denied knowledge to the latter. Second, many ordinary knowledge expressions initially suggest the possibility of non-factive knowledge (Hazlett 2010). For example, someone who improbably survived a plane crash might sensibly say, "I knew I was going to die." However, linguistic analysis and experimental studies suggest that this phenomenon is actually due to other factors, especially perspective-taking (Buckwalter 2014; c.f. Turri 2011). Third, researchers have found that judgments about truth strongly predict knowledge attributions and related judgments, including evaluations of the quality of evidence and propriety of beliefs (Turri 2013a, 2016b).

While all this research is suggestive, it is limited in an important way. In particular, although the behavioral studies compared various cases of true beliefs to false 
beliefs, they did not compare true beliefs to false beliefs that were approximately true. The comparisons were always much starker than that, involving, for example, knowing that a stone is a diamond when it is real but not when it is fake (Turri, Buckwalter, and Blouw 2015: Experiment 4), or knowing the location of an object when it is present but not when it has been stolen and removed (Starmans and Friedman 2012: Experiments 12). Accordingly, although existing evidence supports some sort of conceptual connection between knowledge and truth, it is currently unknown whether that connection takes the form of a necessary condition, such that it rules out knowledge of approximations.

Thus we are led to consider an alternative hypothesis, which is also consistent with this body of evidence: knowledge does not require truth but rather representational adequacy. According this hypothesis, representations could be false but still count as knowledge. Instead, they need only adequately represent, or approximate, the truth. A natural corollary to this hypothesis is that adequacy is partially determined by the purposes of agents in different practical situations. For example, it might be possible to know "the value of pi is 3.14" in a grade-school math classroom, even though it would not be known when this approximation would lead to critically inaccurate objectpositioning over vast distances, such as engineering guidance or landing systems for aircraft.

The representational adequacy account is also motivated by several theoretical and experimental developments in philosophy and cognitive science on the connection between knowledge, action, and practical interests (Fantl and McGrath 2009; Hawthorne 2004; Turri and Buckwalter 2017; Turri, Buckwalter, and Rose 2016). Specifically, this research indicates that judgments about how others should act ("actionability") across 
different situations predict and sometimes directly cause judgments about what others know. In one set of studies, for example, researchers presented participants with short vignettes involving an intelligence analyst who is developing a file on a foreign operative (Turri and Buckwalter 2017: Experiment 1). Researchers found that judgments about how the analyst should act strongly predicted knowledge judgments, and that these actionability judgments were even better predictors of knowledge attribution than judgements regarding what was true about the operative were (Turri, Buckwalter, and Rose 2016). Given that actionability predicts knowledge judgments and that reliance on approximations can be a good way to facilitate action, it is a reasonable conjecture that knowledge attribution will sometimes extend to approximate truths.

This paper presents two experiments that, for the first time, begin testing between the representational adequacy and factivity hypotheses. In experiment 1, we manipulated whether an agent's answer was either false or true (truth-value), and we also manipulated whether the agent's answer was practically adequate to accomplish a salient goal (practical adequacy). Both hypotheses under consideration predict that knowledge attribution will be affected by truth value, but they disagree on the potential effects of practical adequacy. Whereas both accounts can agree that knowledge will be attributed for a true adequate answer, and that knowledge will be denied for a false inadequate answer, the accounts diverge in their predictions for a false adequate answer. The factivity account predicts that knowledge will not be attributed in such a case, whereas the representational adequacy account predicts that it will be. The results supported the representational adequacy account. Experiment 2 replicated the principal finding using a distinct probing method that ruled out an alternative explanation in terms of perspective- 
taking. Overall, then, the results provide initial support for the representational adequacy hypothesis.

Before proceeding, we emphasize that we do not interpret our findings as settling the question in favor of the representational adequacy hypothesis, or conclusively ruling out the factivity hypothesis. We took care to replicate our principal finding and to address, with data, what we take to be the most plausible alternative explanation grounded in previous research on knowledge attributions. Later, in the conclusion, we will also note some limitations and open questions that future research could pursue. But no matter how many times an empirical finding is replicated or how many alternatives are ruled out, there will always be additional alternatives that might be proposed: here as elsewhere, evidence underdetermines theory (Quine 1951). Moreover, when raising questions about an assumption as widely accepted as the factivity hypothesis, it is natural and prudent to be especially cautious before leaping to strong conclusions. Accordingly, we do not view ourselves as having settled our principal research question, and while we provisionally conclude that truth is not a requirement of knowledge, our interpretation of the findings preserves a conceptual link between knowledge and truth (see the conclusion). Instead, our principal accomplishment here is arguably to have clearly identified, based on gaps in existing evidence, an important and previously unaddressed question. Pursuant to that, we view our principal finding as a thought-provoking invitation to others to help think through this set of unresolved issues.

\section{Experiment 1}

\subsection{Method}




\subsubsection{Participants}

Eight hundred twenty-nine participants were tested (aged 19-73 years, mean age $=36$ years; 332 female; 94\% reporting English as a native language). Participants were U.S. residents, recruited and tested online using Amazon Mechanical Turk and Qualtrics, and compensated $\$ 0.30$ for approximately 2 minutes of their time. We chose this recruitment method on the grounds that there is reason to believe that MTurk subjects are more attentive than traditional subject pool participants are (Hauser and Schwarz 2016) and because it has been used extensively in prior work investigating both knowledge representation generally (e.g. Starmans and Friedman 2012; Turri, Buckwalter, and Blouw 2015; Turri, Friedman, and Keefner 2017; Turri and Park 2018), and the factivity of knowledge specifically (e.g. Buckwalter 2014; Turri 2018). Repeat participation was prevented within and across experiments. After testing, participants completed a brief demographic questionnaire. The same recruitment and compensation procedures were used for all experiments reported in this paper.

\subsubsection{Materials and procedure}

Participants were assigned to one of twenty conditions in a 2 (truth-value: true, false) $x 2$ (adequacy: strict, lenient) x 5 (scenario: war, caribou, ticket, vision, foundation) design. The truth-value factor manipulated whether the answer the protagonist gave in the story was true or false. The adequacy factor manipulated whether a practically successful answer required strict true (strict) or provided a margin of error (lenient). The scenario factor manipulated what question the agent was considering, and other features of her situation. We included this factor to support generalization of the results beyond the specific stimuli studied here (Clark 1973; Baayen, Davidson and Bates 2008; Judd, 
Westfall and Kenny 2012). All stimuli used in this study are included in a supplemental file. To give readers a sense of the materials, we include one scenario and the test statements here, with the truth-value and adequacy manipulations shown in brackets:

Drew is a civil engineer designing a structure. To fit, he must use the length of an existing foundation, to [the exact inch/within 10 inches] of its total length. After thinking carefully, Drew uses [9905/9910] inches. The actual length of the foundation was 9910 inches.

Participants then evaluated the following four test items:

1. What was the actual length of the foundation? (comprehension)

2. The length Drew used was technically false. (truth, reverse coded)

3. The length Drew used was adequate enough to fit. (adequacy)

4. Drew knows the length of the foundation. (knows)

Responses to the comprehension item were collected using randomly rotated fixed choices. Responses to truth, adequacy, and knows items were collected on a standard 7point Likert scale, 1 ("strongly disagree") - 7 ("strongly agree"), left-to-right on the participant's screen.

\subsection{Results}

Twenty-three participants failed the comprehension question and were excluded from the analysis. No other exclusions occurred. For the purposes of analysis, scores on the truth item were reverse-coded so that higher scores indicated higher truth judgments.

We conducted a linear mixed effects analysis on the knows item. We included as fixed effects truth-value, standard, an interaction of truth-value and standard, participant sex, participant age, and reported political preference. We included a random intercept for 
scenario and random slopes with respect to truth-value and standard. Regarding the random effects, the random intercept for scenario and the random slope with respect to standard were insignificant, but the random slope with respect to truth-value was significant (see Table 1). The significant random slope occurred because in the war scenario, the agent's answer being false depressed knowledge attributions more than for the other scenarios (see Figure 1). Regarding the fixed effects, biological sex, age, and political ideology were insignificant (see Table 2). There was a main effect of truth. There was no main effect of standard, but it entered into a significant interaction with truth-value. 
(A) Judgments of Practical Adequacy, Knowledge, and Truth

Subjects made all three judgments. Dots overlay distributions and show means with $95 \%$ confidence intervals.
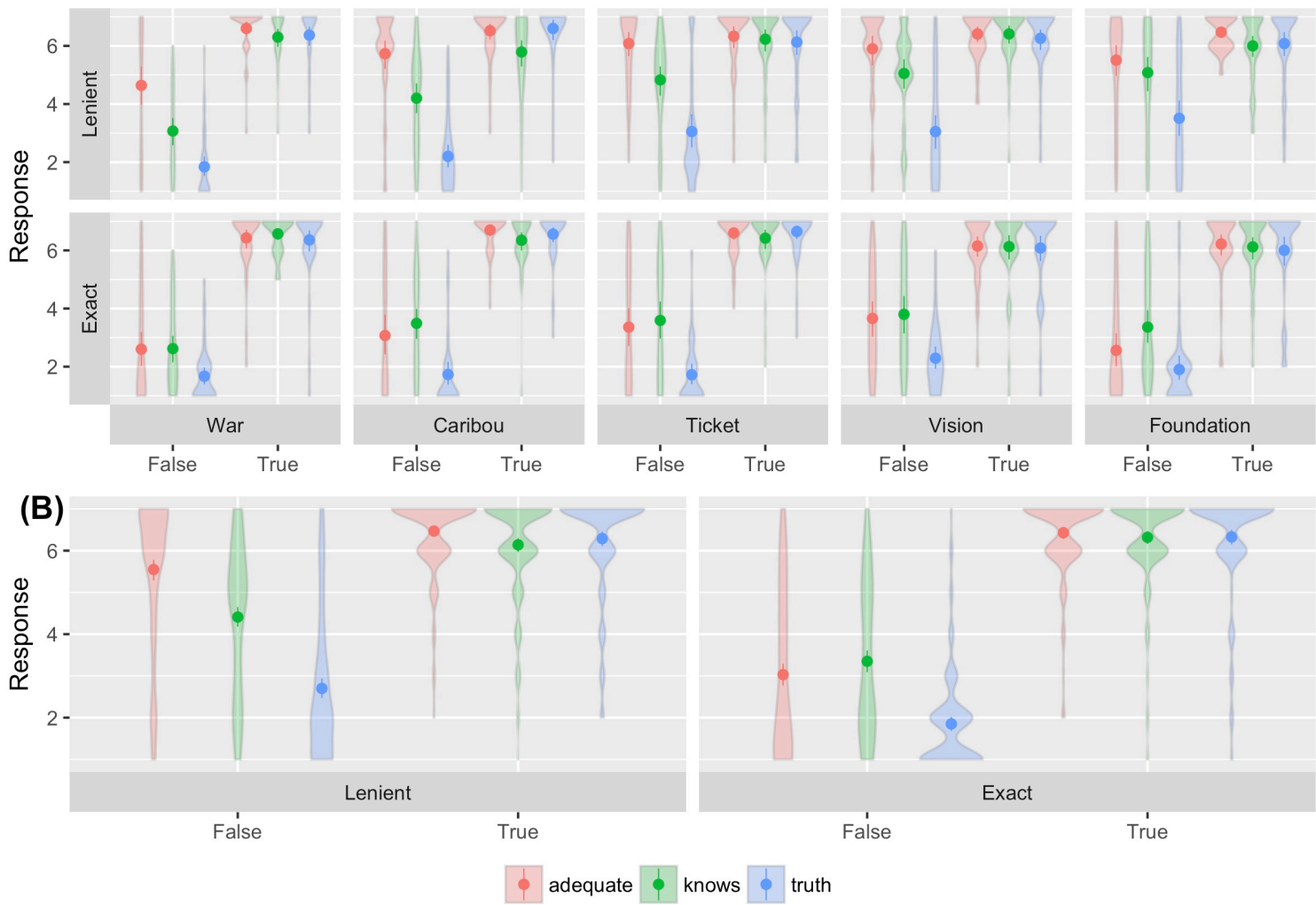

Figure 1. Experiment 1. Mean response to test statements concerning whether an answer was adequate, known, and truth (shown reverse coded). Scales ran 1 (SD) - 7 (SA). Panel A shows means broken down by truth-value (false, true), standard (lenient, exact), and scenario (five variants). Panel B shows the same data collapsed across scenario, which was not of independent theoretical interest.

Table 1. Experiment 1. Likelihood ratio tests for the random effects in the linear mixed analysis.

\begin{tabular}{llllll}
\hline term & log. lik. & AIC & LRT & df & p \\
\hline Scenario & -1469.56 & 2977.12 & $<.001$ & 1 & $>.999$ \\
Truth-value | Scenario & -1485.22 & 3004.44 & 31.323 & 3 & $<.001$ \\
Standard | Scenario & -1471.30 & 2976.61 & 3.489 & 3 & .322 \\
\hline
\end{tabular}


Table 2. Experiment 1. F tests for the fixed effects in the linear mixed analysis.

\begin{tabular}{llllll}
\hline term & sum squares & df1 & df2 & F & p \\
\hline Truth-value & 104.328 & 1 & 4.028 & 48.676 & .002 \\
Standard & 14.797 & 1 & 4.092 & 6.904 & .057 \\
sex & 2.952 & 1 & 786.844 & 1.377 & .241 \\
age & 0.909 & 1 & 787.688 & 0.424 & .515 \\
politics & 9.203 & 6 & 787.181 & 0.716 & .637 \\
Truth-value*Standard & 76.430 & 1 & 786.168 & 35.660 & $<.001$ \\
\hline
\end{tabular}

Follow-up independent samples t-tests revealed that the truth-value/standard interaction occurred because knowledge attribution did not differ between true lenient (M $=6.14, \mathrm{SD}=1.20)$ and exact lenient $(\mathrm{M}=6.32, \mathrm{SD}=1.10)$ conditions, $\mathrm{t}(401)=-1.58, \mathrm{p}$ $=.11$, but it was significantly higher in false lenient conditions $(\mathrm{M}=4.41, \mathrm{SD}=1.82)$ than in false exact conditions $(M=3.35, S D=1.82), t(396)=5.79, p<.001, d=0.58$. Mean knowledge attribution was significantly above the neutral midpoint $(=4)$ in false lenient conditions, $\mathrm{t}(202)=3.20, \mathrm{p}=.001, \mathrm{~d}=0.22$, whereas it was significantly below the midpoint in false exact conditions, $\mathrm{t}(195)=-4.97, \mathrm{p}<.001, \mathrm{~d}=0.36$. This same pattern was also reflected in median knowledge attribution, which was "slightly agree" (= $5)$ in false lenient conditions and "slightly disagree" $(=3)$ in false exact conditions.

The preceding analyses provide evidence that the independent variables affected knowledge attributions in the way predicted by the representational adequacy account. 
However, that prediction was based on assumptions about how specific manipulations would affect participants' interpretation of the case. In particular, it assumes that the truth-value manipulation affects how participants judge the relevant propositions truthvalue, and that the standard manipulation affects how participants judge the practical adequacy of the agent's answer. Although these assumptions are reasonable, questioning them can also be reasonable. Accordingly, rather than rely solely on our interpretation of the independent variables, we conducted a multiple linear regression analysis to predict knowledge attributions based on participants' own judgments about truth-value and practical adequacy.

The model included response to the truth and adequacy items and assignment to scenario condition as predictors, and it included response to the knows item as outcome. The regression model was significant and explained 59\% of the variance in knowledge attributions (see Table 3). Participant response to the truth and adequacy items each made a unique, statistically significant contribution, and the magnitude of their contributions was comparable ( $\beta$ values of .344 for truth and .386 for adequacy). Scenario was also significantly predictive. 
Table 3. Experiment 1. Multiple linear regression analyses predicting knowledge attributions.

\begin{tabular}{|c|c|c|c|c|c|}
\hline model & term & estimate & std. error & $\mathbf{t}$ & $\mathbf{p}$ \\
\hline \multirow[t]{8}{*}{ Full } & Intercept & 1.284 & 0.147 & 8.744 & $<.001$ \\
\hline & truth & 0.344 & 0.024 & 14.240 & $<.001$ \\
\hline & adequate & 0.386 & 0.029 & 13.158 & $<.001$ \\
\hline & \multicolumn{5}{|l|}{ Scenario } \\
\hline & Caribou & 0.077 & 0.137 & 0.564 & .573 \\
\hline & Ticket & 0.317 & 0.139 & 2.279 & .023 \\
\hline & Vision & 0.414 & 0.140 & 2.945 & .003 \\
\hline & Foundation & 0.348 & 0.139 & 2.509 & .012 \\
\hline \multirow[t]{3}{*}{ Reduced } & Intercept & 1.486 & 0.126 & 11.767 & $<.001$ \\
\hline & truth & 0.346 & 0.024 & 14.284 & $<.001$ \\
\hline & adequate & 0.388 & 0.029 & 13.237 & $<.001$ \\
\hline
\end{tabular}

Note. Full model: $\mathrm{F}(6,799)=192.30, \mathrm{p}<.001, \mathrm{R}^{2}=.591$. Reference class for scenario: war. Reduced model: $\mathrm{F}(2,803)=563.50, \mathrm{p}<.001, \mathrm{R}^{2}=.584$

We included scenario because it was significant in the mixed effects model (reported above). However, it was not of independent theoretical interest, so we ran another multiple regression model without it, which included only participant response to the truth and adequate items as predictors. The difference between the full model (including scenario) and this reduced model (omitting scenario) was statistically significant, $\mathrm{F}(4)=3.39, \mathrm{p}=.009$. But a comparison of the $\mathrm{R}^{2}$ values revealed that the difference was very small: the full model explained $59.1 \%$ of the variance in knowledge attribution, whereas the reduced model explained 58.4\% (see Table 3). Responses to the 
truth and adequacy items were again both significant and roughly comparable in their magnitude ( $\beta$ values of .346 for truth and .388 for adequacy).

\subsection{Discussion}

The results indicate that truth evaluations have a strong effect on knowledge attributions. Knowledge attribution was much higher when a proposition was true than when it was false. The results also indicate that evaluations of practical adequacy can have a comparable effect on knowledge attributions. When an agent's answer was false, knowledge attribution was much higher if the answer was practically adequate rather than practically inadequate. When a false answer was practically inadequate, participants tended to deny knowledge, but when it was practically adequate, they tended to attribute knowledge. Even though the central tendency was to attribute knowledge for a practically adequate false answer, knowledge attribution was still significantly higher for a practically adequate true answer. Overall, then, the results suggest that although truth is not viewed as a requirement of knowledge, thereby supporting the representational adequacy account, truth probably is a feature of paradigmatic knowledge, thereby potentially revealing a kernel of truth in the factivity account.

\section{Experiment 2}

Experiment 1 measured knowledge attribution by measuring agreement or disagreement with a single knowledge statement. However, prior research suggests that some knowledge attributions concerning false representations should not be interpreted literally but rather as indicating how things seem from the agent's perspective (Buckwalter 2014). In other words, sometimes participants use a knowledge statement in order to 
perspective-take, effectively substituting one task for another. This raises the possibility that when participants in experiment 1 attributed knowledge of false approximations, this should be interpreted as perspective-taking: from the agent's perspective, it seemed like he knew. The present experiment was designed to evaluate this possibility by using a different probe that disentangles perspective-taking from knowledge attribution.

\subsection{Method}

\subsubsection{Participants}

Four-hundred and five new participants were tested (aged 18-73 years, mean age $=34$ years; 187 female; 97\% reporting English as a native language).

\subsubsection{Materials and procedure}

Participants were assigned to one of four conditions in a 2 (truth-value: true, false) x 2 (adequacy: strict, lenient) between-subjects design. Because we switched from a 7-point scaled measure to a dichotomous measure (see below), we increased the sample size to 100 participants per condition to ensure adequate power. The scenarios were taken verbatim from the ticket condition in Experiment 1. After reading a scenario, all participants were presented with a single test item designed to disentangle literal knowledge attribution from perspective taking (for a related procedure, see Buckwalter 2014; for precedents and discussion, see Rose et al. 2019; Starmans and Friedman 2012; Turri 2014; Weinberg, Nichols, and Stich 2001). Critically, the response options do not force participants to nominally attribute knowledge as a substitute for acknowledging the agent's perspective.

1. Drew ___ the cost of the ticket. (knowledge) 
To complete this item, participants could select only one of the following two options: "knows" or "only thinks he knows". These answer choices were randomly rotated and presented below the test item while the story remained on top of the screen. After completing this item, participants were taken to a new screen and completed a short demographic questionnaire.

\subsection{Results}

A binary logistic regression revealed that both main effects and an interaction effect between adequacy and truth predicted response to the knowledge test item (see Table 16).

Table 4. Experiment 2. Logistic regression predicting responses to the Projection item. The full model was statistically significant, $\mathrm{X}^{2}(3, \mathrm{~N}=405)=95.74, p<.001$, and explained between $21 \%$ and $30 \%$ variance in response to the test item

\begin{tabular}{lccccccc}
\hline Predictor & B & SE & Wald & df & p & Odds Ratio & 95\% CI \\
\hline Truth & 2.85 & 0.39 & 52.47 & 1 & $<.001$ & 17.27 & $7.99,37.34$ \\
Adequacy & 1.13 & 0.29 & 14.68 & 1 & $<.001$ & 3.08 & $1.73,5.47$ \\
T*A & -1.44 & 0.53 & 7.29 & 1 & .007 & 0.24 & $0.08,0.67$ \\
Constant & -0.62 & 0.21 & 8.72 & 1 & .003 & 0.54 & \\
\hline
\end{tabular}

The "knows" response was selected at rates exceeding chance in the true strict and true lenient conditions $(90 / 87 \%$ : binomial tests, $p \mathrm{~s}<.001$, test proportions $=.50)$. Most importantly, the "knows" response was also selected at rates exceeding chance in the false lenient condition $(62 \%$ : binomial test, $p<.001$, test proportion $=.50)$. This significantly differed from response in the strict false condition, $X^{2}(1, N=201)=15.07$, $p<.001$, Cramer's $\mathrm{V}=.274$, in which “only thinks he knows" was selected at rates exceeding chance $(65 \%$ : binomial test, $p<.001$, test proportion $=.50)$. 


\subsection{Discussion}

These results replicate the findings of experiment 1 using a different probing method designed to test an alternative explanation due to perspective-taking. The results undermine the alternative explanation and provide further support for the representational adequacy account. Participants again attributed knowledge of adequate approximate truths but denied it for inadequate approximate truths.

\section{General Discussion}

Does knowledge require truth, or is it possible to know some falsehoods that are adequate for present purposes? According to the factivity account, knowledge requires truth, whereas according to the representational adequacy account, a false but practically adequate representation can suffice. We conducted two experiments to begin investigating which of these two accounts better fits the ordinary knowledge concept. We presented participants with simple, realistic scenarios and manipulated the truth and adequacy of an answer, so that the two competing accounts made clear and opposed predictions. Although both accounts predict that knowledge attribution will be affected by an answer's truth value, they disagree on the potential effect of an answer's practical adequacy. More specifically, whereas both accounts can agree that knowledge will be attributed for a true adequate answer, and both accounts can agree that knowledge will be denied for a false inadequate answer, the accounts disagree on whether knowledge will be attributed for a false adequate answer. The factivity account predicts that knowledge will not be attributed in such a case, whereas the representational adequacy account predicts that it will be. 
The findings confirm this latter prediction, supporting the representational adequacy account. Across different narrative contexts, types of approximation, and adequacy conditions, participants agreed that some answers, although false, count as knowledge when they are adequate to accomplishing certain objectives, such as winning a prize, getting a job, or building a foundation (experiment 1). This finding is not explained by perspective-taking (experiment 2). Overall, then, our findings support the conclusion that it is conceptually possible for some false representations to qualify as knowledge.

The representational adequacy account links knowledge to pragmatic success and predicts that knowledge is possible when representations are adequate enough for practical purposes. If it is conceptually possible for false but practically adequate representations to count as knowledge, then we should expect this to be reflected in the knowledge attributions of competent language users, which is what we observed in the two experiments reported here. However, an alternative interpretation of the data is that the key findings were instead due to task demand. For example, one could propose that it is conceptually impossible to know false propositions, regardless of their practical adequacy, but participants in the relevant conditions were led to attribute false knowledge because the stimuli stipulated practically acceptable margins for error. Absent that stipulation, the alternative goes, participants would not have treated practical adequacy as relevant, at least not to the extent that it would sustain a knowledge attribution in the absence of truth.

This is an interesting possibility worth considering, and we think that existing findings render it unlikely. First, if task demand led participants to attribute knowledge in 
this way, then presumably it would also lead them to evaluate the underlying propositions as true too. In other words, if participants are sensitive and accommodating regarding their knowledge attributions in this way, then they probably will do the same with their truth judgments. However, this is not what we observed. Instead, participants tended to attribute knowledge but deny truth. Second, prior research has found a strong connection between judgments about knowledge and judgments about how agents should act, even when the stimuli did not stipulate whether the underlying proposition was true, mention margins for error, or stipulate standards of practical adequacy (Turri, Buckwalter and Rose 2016; see also Turri and Buckwalter 2017). The representational adequacy hypothesis can explain these previous findings, whereas the task demand hypothesis cannot. Of course, it is theoretically possible that the task demand hypothesis is correct about the findings from the present studies, and the previous findings, which also suggest an important connection between knowledge and practice, are misleading for completely separate reasons. Further work would be required to vindicate this more complicated proposal. In the meantime, we submit that the representational adequacy account is theoretically preferable to the task demand hypothesis.

The present findings have theoretical significance by furthering our understanding of the conceptual connection between knowledge and truth. This theoretical advance is made more significant by the fact that knowledge attributions play several important roles in ordinary social cognition, including in predicting (Turri 2017) and evaluating (Turri 2016a; Turri, Friedman, and Keefner 2017) others' behavior. Many leading philosophers agree that knowledge requires truth (see the introduction for references). But there has been relatively little research examining the nature of the connection between knowledge 
and truth, especially whether approximately true representations can be known. In light of the present findings, it seems likely that approximations in scientific measurement, scholarly inquiry, and everyday decision making are often thought to qualify as knowledge.

Consistent with this, it is possible that truth is essential to the ordinary knowledge concept, in the following way. The ordinary knowledge concept might be a prototype concept (Rosch and Mervis 1975; Wittgenstein 1953). Instead of comprising a "checklist" of necessary and sufficient conditions (Fillmore 1975), the knowledge concept might summarize the central tendency of representations that constitute knowledge. This central tendency consists of a cluster of properties that prototypical knowledge has. Some of these properties matter more, are more central, to the category. An instance of knowledge similar to the prototype is typical but an instance dissimilar to the prototype is atypical, just as a sparrow is typical of birds but an ostrich is atypical. Suppose that prototypical knowledge is true, rather than merely approximately true. It follows that a merely adequate approximation cannot be typical in the fullest sense. More ambitiously, suppose that truth is a centrally important feature of the prototype, akin to flight for birds. It follows that a merely adequate approximation could always be viewed as atypical, which in turn could explain some of the intuitive motivation for the factivity account among philosophers.

This prototype hypothesis is arguably the most conservative interpretation of the data that rejects the factivity hypothesis. Additionally, it would also explain why we observed a significant difference in our studies between how participants judged adequate representations that were true compared to merely approximately true. Even though the 
central tendency was to attribute knowledge for both, people were more confident in this attribution for truths than for approximate truths, which is the pattern one would predict based on the prototype hypothesis. Future research could investigate further predictions of this hypothesis by testing, for example, whether knowledge attributions are reliably faster for true representations than for approximately true ones.

The findings may also have social and political significance. It is often said that we are living in a "post-truth" age in which personal interests shape what is believed to be true. We observed that participants were reliably willing to attribute knowledge for some approximate truths but not others. In particular, when the agent's answer was adequate for a salient practical purpose, participants tended to attribute knowledge, whereas they tended to deny knowledge when the answer was practically inadequate. This suggests a possible alternative mechanism underlying "post-truth" disagreements rooted in folk epistemology: it could be that disagreements are not caused by differences of opinion about what is true per se, but rather by differences of opinion about what is known, due to judgments about what is adequate. Future research is needed to test this hypothesis in greater detail.

Future research could also expand on the present results in several ways. For example, further research could study the specificity of approximate knowledge attribution. The current research demonstrates that an approximation can suffice for knowing the answer to a question, such as knowing the cost of a ticket. However, it would also be informative to learn whether participants attribute knowledge at similar rates for more specific representations, such as knowing the ticket costs a particular amount. Future research might also explore the integration of approximate knowledge 
with other kinds of representations, such as representations of belief. One possibility is that approximate knowledge is attributed when no corresponding belief is attributed, further contributing a more nuanced understanding of the conceptual connections between knowledge, truth, and belief.

In conclusion, we emphasize that our findings do not conclusively rule out the factivity account or definitively settle the matter in favor of the representational adequacy account. It is a live possibility that future research will yield results that undermine the provisional conclusions we arrived at here. We welcome and encourage such research, as it would help advance our collective understanding of the underlying issues. In the meantime, we submit that the conceptual connection between knowledge and truth is a topic worthy of serious attention from the research community. As noted in the introduction, the factivity account, despite being a widespread assumption with some support from previous studies, had not faced the strongest test of its mettle. The strongest test would not compare a strictly true answer to a false, practically inadequate answer that is very far from the truth. A much stronger test involves comparing a strictly true answer to a false but approximately true, practically adequate answer.

Acknowledgments - [[omitted at review stage $]]$.

\section{References}

Audi, Robert (1998), Epistemology: A Contemporary Introduction to the Theory of Knowledge (New York: Routledge). 
Blouw, Peter, Buckwalter, Wesley, and Turri, John (Forthcoming), 'Gettier Cases: A Taxonomy', in R. Borges, C. de Almeida, and P. Klein (eds.), Explaining Knowledge: New Essays on the Gettier Problem (Oxford: Oxford University Press).

BonJour, Laurence (2002), Epistemology: Classic Problems and Contemporary Responses (New York: Rowman \& Littlefield).

Buckwalter, Wesley (2014), 'Factive Verbs and Protagonist Projection', Episteme, 11 (4), 391-409.

Chisholm, Roderick (1989), Theory of Knowledge (Englewood Cliffs, New Jersey: Prentice Hall).

Davidson, Donald (2001), Subjective, Intersubjective, Objective (Oxford: Clarendon Press).

Fantl, Jeremy and McGrath, Matthew (2009), Knowledge in an Uncertain World (New York: Oxford University Press).

Feldman, Richard (2003), Epistemology (Upper Saddle River, New Jersey: Prentice Hall).

Fillmore, Charles J. (1975), 'An Alternative to Checklist Theories of Meaning', 1975, 9.

Fumerton, Richard (2006), Epistemology (Malden, MA: Blackwell).

Greco, J (2010), Achieving Knowledge: A Virtue-Theoretic Account of Epistemic Normativity (Cambridge: Cambridge University Press).

Hauser, David J. and Schwarz, Norbert (2016), 'Attentive Turkers: Mturk Participants Perform Better on Online Attention Checks Than Do Subject Pool Participants', Behavior Research Methods, 48 (1), 400-07. 
Hawthorne, John (2004), Knowledge and Lotteries (Oxford: Oxford University Press).

Hazlett, Allan (2010), 'The Myth of Factive Verbs', Philosophy and Phenomenological Research, 80 (3), 497-522.

Mittal, Sparsh (2016), 'A Survey of Techniques for Approximate Computing', ACM Comput. Surv., 48 (4), 1-33.

Nagel, Jennifer, Juan, Valerie San, and Mar, Raymond A (2013), 'Lay Denial of Knowledge for Justified True Beliefs', Cognition, 1-10.

NASA/JPLedu (2017), 'How Many Decimals of Pi Do We Really Need?', $<$ https://www.jpl.nasa.gov/edu/news/2016/3/16/how-many-decimals-of-pi-do-wereally-need/>, accessed September 22, 2017.

Pritchard, Duncan (2006), What Is This Thing Called Knowledge? (New York: Routledge).

Rosch, Eleanor and Mervis, Carolyn B. (1975), 'Family Resemblances: Studies in the Internal Structure of Categories', Cognitive Psychology, 7 (4), 573-605.

Rose, David, et al. (2019), 'Nothing at Stake in Knowledge', Noûs, 53 (1), 224-47.

Sandoval, William A., Greene, Jeffrey A., and Bråten, Ivar (2016), 'Understanding and Promoting Thinking About Knowledge:Origins, Issues, and Future Directions of Research on Epistemic Cognition', Review of Research in Education, 40 (1), $457-$ 96.

Sartwell, Crispin (1992), 'Why Knowledge Is Merely True Belief', The Journal of Philosophy, 89 (4), 167-80.

Sosa, Ernest (1991), Knowledge in Perspective (Cambridge: Cambridge University Press). 
Starmans, Christina and Friedman, Ori (2012), 'The Folk Conception of Knowledge', Cognition, 124 (3), 272-83.

Turri, J, Friedman, O, and Keefner, A (2017), 'Knowledge Central: A Central Role for Knowledge Attributions in Social Evaluations', Q J Exp Psychol (Hove), 70 (3), $504-15$.

Turri, John (2011), 'Mythology of the Factive', Logos \& Episteme, 2 (1), 143-52.

Turri, John (2013a), 'The Test of Truth: An Experimental Investigation of the Norm of Assertion', Cognition, 129 (2), 279-91.

Turri, John (2013b), 'A Conspicuous Art: Putting Gettier to the Test', Philosophers' Imprint, 13 (10), 1-16.

Turri, John (2014), 'The Problem of Esee Knowledge', Ergo, 1 (4), 101-27.

Turri, John (2016a), Knowledge and the Norm of Assertion: An Essay in Philosophical Science (Cambridge, UK: Open Book Publishers).

Turri, John (2016b), 'The Radicalism of Truth-Insensitive Epistemology: Truth's Profound Effect on the Evaluation of Belief', Philosophy and Phenomenological Research, 93 (2), 348-67.

Turri, John (2016c), 'A New Paradigm for Epistemology from Reliabilism to Abilism', Ergo, an Open Access Journal of Philosophy, 3.

Turri, John (2017), 'Knowledge Attributions and Behavioral Predictions', Cognitive Science, 41 (8), 2253-61.

Turri, John (2018), 'Revisiting Norms of Assertion', Cognition, 177, 8-11. http://doi.org/10.1016/j.cognition.2018.03.023 
Turri, John and Buckwalter, Wesley (2017), 'Descartes's Schism, Locke's Reunion: Completing the Pragmatic Turn in Epistemology', American Philosophical Quarterly, 54 (1), 25-46.

Turri, John and Park, YeounJun (2018), 'Knowledge and Assertion in Korean', 42 (6), 2060-80.

Turri, John, Buckwalter, Wesley, and Blouw, Peter (2015), 'Knowledge and Luck', Psychonomic Bulletin and Review, 22, 378-90.

Turri, John, Buckwalter, Wesley, and Rose, David (2016), 'Actionability Judgments Cause Knowledge Judgments', Thought, 5 (3), 212-22.

Weinberg, Jonathan M, Nichols, Shaun, and Stich, Stephen (2001), 'Normativity and Epistemic Intuitions', Philosophical Topics, 29 (1-2), 429-60.

Williamson, Timothy (2000), Knowledge and Its Limits (Oxford: Oxford University Press).

Wittgenstein, Ludwig (1953), Philosophical Investigations, trans. G. E. M. Anscombe (Oxford: Basil Blackwell).

Zagzebski, Linda T. (2009), On Epistemology (Belmont, CA: Wadsworth). 